POETRY

\title{
First time
}

They say you never forget your first time and I've learned that this is true.

Once in a while, my mind wanders back to a night, now years away,

The memory triggered by something ever so slight,

A sound, a scent, a voice.

And I remember clearly.

I see his lovely body, the chest exposed, sweat trickling down the well-formed chest.

I see his hands. A working man's hands,

Encrusted with the remains of a day on the construction site. His arms, tattooed, declaring unfailing love for an old girlfriend.

I smell his breath, tinged with beer and cigarettes and the bar from which he had been

brought.

I hear that breath, rasping, desperate. And pleading. I hear panic in the voice of the anesthetist resident, A difficult intubation.

I see, the blood-stained drool that oozes down his unshaved jaw.

I hear, the crack of the defibrillator on that lovely chest.

Crack. Quiet. Again. Again. AGAIN FOR GOD'S SAKE.

And then I hear what we all hear sooner or later.

The silence.

The Team stands still, in respect or shame, I am not sure which.

I hear the rubbery sound of gloves being removed.

And that, more than anything else, signals that it is finished.

This memory, not unpleasant, is one I hold on to.

My first death.

I was there to watch this young man die. So it's okay that I am visited from time to time

in this way.

As always, the memory fades quickly.

I am comforted though, knowing that in weeks, months, years, it will return to me.

Triggered by something ever so slight.

\section{Eileen P. Sloan MD}

Department of Psychiatry

Mount Sinai Hospital

Toronto, Ont.

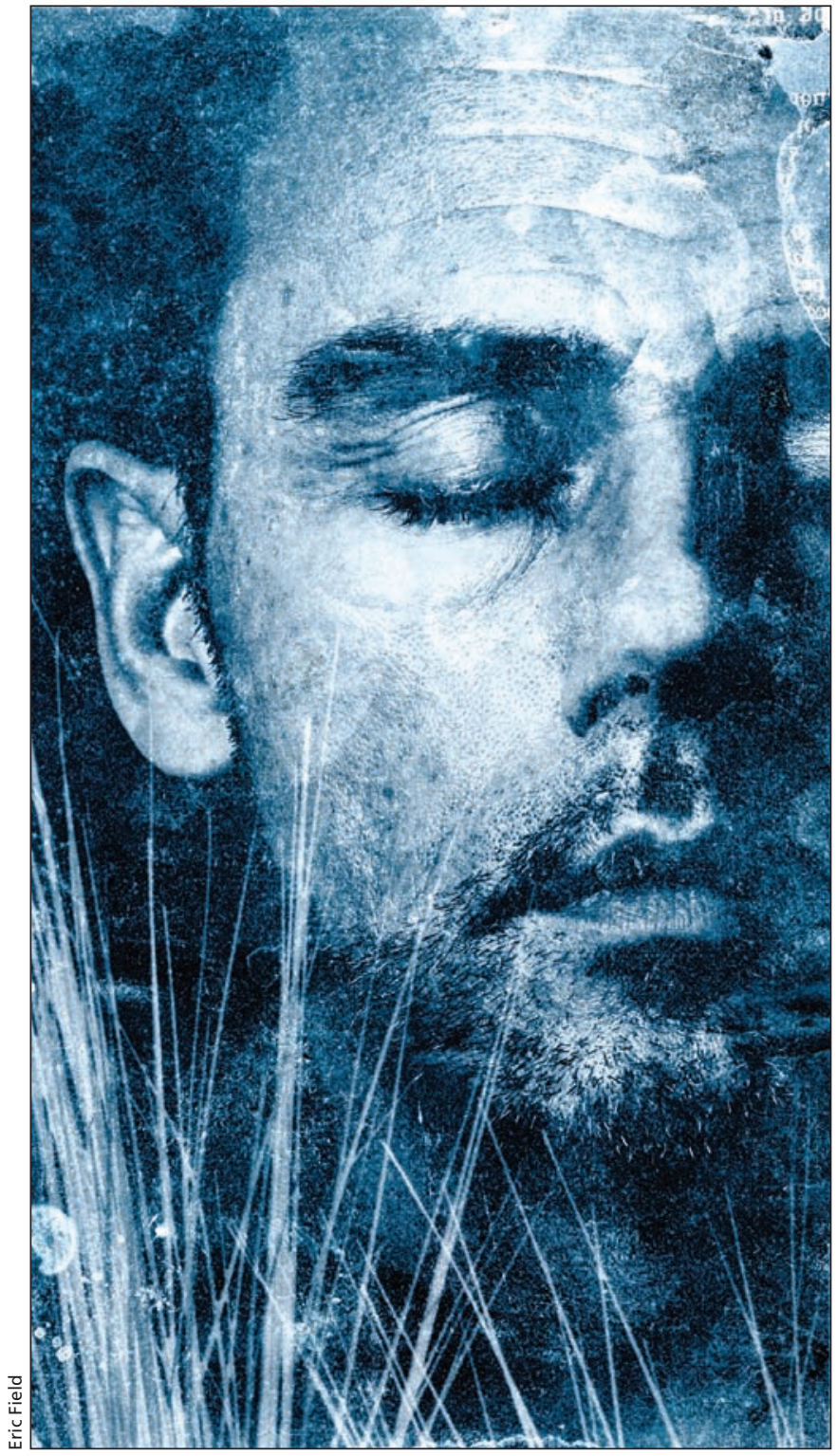

"First time" won first prize in the poetry category of the 2012 ARS Medica and CMAJ Humanities Poetry and Prose contest.

CMAJ 2013. DOI:10.1503/cmaj.120817 